\title{
UN ESTUDIO CUALITATIVO ACERCA DE LA PERCEPCIÓN DEL CARÁCTER IRREEMPLAZABLE DE LA PERSONA
}

\author{
A QUALITATIVE STUDY ON THE PERCEPTION OF THE \\ IRREPLACEABLE CHARACTER OF A PERSON
}

\author{
Juan F. Franck* \\ Universidad Austral \\ Buenos Aires-Argentina \\ Lucas Marcelo Rodriguez \\ CONICET-Universidad Católica Argentina \\ Buenos Aires-Argentina

\section{Mariano Asla} \\ Universidad Austral \\ Buenos Aires-Argentina \\ Recibido febrero de 2021/Received February, 2021 \\ Aceptado junio de 2021/Accepted June, 2021
}

\begin{abstract}
RESUMEN
El objetivo del presente estudio interdisciplinar es explorar la comprensión del carácter irreemplazable del ser personal en adultos. Para ello se entrevistó a diez adultos de entre 25 y 46 años de ambos sexos, que estuviesen en pareja por un tiempo mínimo de 1 año (teniendo en cuenta que la pareja es un vínculo significativo con otra persona). Se realizaron varias entrevistas para recoger sus reacciones y comentarios acerca de un capítulo de ficción cuya trama se desenvuelve alrededor de la posibilidad de reemplazar una persona por un software-robot ("Regresa enseguida", de Black Mirror). Las categorías surgidas en el análisis cualitativo fueron: shock, realidad-irrealidad, irreemplazable e intuición. El artículo discute dichos hallazgos a la luz de un aspecto del problema filosófico de la identidad personal. El análisis sugiere que existe una percepción intuitiva fuerte del carácter propiamente individual de cada persona y, por tanto, de su irreemplazabilidad. Esta percepción robusta, sin embargo, no se manifestó en una claridad pareja a la hora de definir con exactitud cuál es la raíz o fundamento de esa identidad. Aunque ciertamente no resuelve el debate, en nuestra interpretación esta observación otorga plausibilidad a la postura denominada enfoque simple, que sostiene que la identidad personal es un hecho primitivo, no reductible a cualidades físicas ni mentales. Señalamos también una posible continuación del estudio.

Palabras Claves: Identidad personal, enfoque simple, psicología de la personalidad, relaciones interpersonales, experimentos mentales, filosofía experimental.
\end{abstract}

\section{ABSTRACT}

The present interdisciplinary study aims to explore our understanding of the irreplaceable character of the person among adults. We interviewed 10 adults between 25 and 46 years old, of both sexes, who had been living together with a partner for at least 1 year (considering that a relationship is expected to be a significant bond). We carried out a series of interviews to collect their reactions and comments on a fictional episode whose plot addresses the possibility of replacing a person with a software or a robot (episode "Be Right Back", Black Mirror). The categories that emerged from the qualitative analysis were: shock, reality-unreality, irreplaceable, intuition. The article discusses these findings in light of one aspect of the philosophical problem of the personal 
identity. The analysis suggests that there is a strong intuitive perception of the character of each person and, therefore, they are irreplaceable. However, this robust perception was not accompanied by an equally clear justification when it came to define the root or base of such identity. Even though it certainly does not solve the debate, in our interpretation this conveys plausibility to the simple approach, which argues that personal identity is a primitive fact, non-reducible to physical or mental qualities. We also point out a possible continuation of this study.

Key Words: Personal identity, simple view, psychology of personality, interpersonal relationships, mental experiments, experimental philosophy.

\section{AGRADECIMIENTOS}

Agradecemos a tres lectores anónimos sus agudas recomendaciones, que contribuyeron sustancialmente a mejorar una primera versión del artículo. Esta investigación fue posible gracias a un subsidio del proyecto TheoPsych de Blueprint 1543. Las opiniones expresadas corresponden a los autores y no necesariamente reflejan la visión de Blueprint o del proyecto TheoPsych.

\section{INTRODUCCIÓN}

El presente estudio tiene como objetivo principal explorar la comprensión de la identidad del ser personal en personas adultas. Su carácter es interdisciplinar, ya que su marco conceptual es el denominado problema filosófico de la identidad personal, que abarca diversas discusiones bajo un mismo denominador. Algunas de ellas son: ¿qué es ser persona?, ¿qué somos fundamentalmente?, ¿quién soy? o ¿quiénes somos?, ¿qué nos hace ser quienes somos?, ¿cuáles son las condiciones de nuestra persistencia en el tiempo?, ¿qué es lo verdaderamente importante en esa persistencia?, etc. (Olson, 2021; Muñoz Corcuera, 2020). Las discusiones pueden asumir un carácter metafísico, intentando comprender en qué consiste propiamente ser persona -tanto en general como esta persona particular- o epistemológico, que inquiere acerca de cómo reconocemos a una persona o cómo sabemos que es la misma persona. Por el problema de la identidad personal se entiende actualmente sobre todo la cuestión de la persistencia, es decir el problema diacrónico, que busca determinar qué hace que una persona sea la misma persona a lo largo del tiempo. La cuestión sincrónica consistiría en cambio en dilucidar qué hace que una persona sea la persona que es. Ambas aristas del problema -la sincrónica y la diacrónica- hacen a la dimensión metafísica del problema y de ellas dependerá en parte la respuesta a la cuestión epistemológica.

Por su objeto y por su método, nuestro estudio puede entenderse también en el marco de la llamada "filosofía experimental", que ha alcanzado una caracterización más o menos precisa en el último decenio y medio, aunque ha recibido también algunas críticas (Knobe y Nichols, 2017; Plakias, 2015). La filosofía experimental consiste principalmente en explorar las intuiciones de la gente sobre temas de interés para la filosofía, empleando métodos propios de las ciencias sociales. El ánimo de estos estudios no es probar o refutar una determinada tesis filosófica, sino conocer mejor las ideas de las personas.

Presentaremos primero sintéticamente algunas de las principales posiciones en la discusión filosófica respecto de la identidad personal, añadiendo algunos aportes desde la Psicología e indicando más específicamente la motivación del estudio y sus objetivos. La presentación será forzosamente breve y con el fin de enmarcar la investigación, no de discutir exhaustivamente el problema filosófico en sí mismo, y mucho menos de decidirlo ni de adoptar y defender una posición propia. Describiremos luego el estudio realizado, indicando la metodología seguida y los resultados obtenidos. Finalmente haremos un análisis de esos resultados, señalando además tanto sus limitaciones como un posible desarrollo ulterior de la investigación.

\section{EL PROBLEMA FILOSÓFICO Y PSICOLÓGICO DE LA IDENTIDAD PERSONAL}

Aunque el tema es de los más discutidos en la historia de la filosofía, en su expresión más reciente parte del modo en que fue conceptualizado por John Locke y de la discusión inmediatamente posterior, que tuvo como protagonistas a David Hume, Joseph Butler y Thomas Reid (Perry, 2008), entre otros. Las posiciones actuales más sofisticadas se remiten también a algunos de esos filósofos (Córdoba, 2017; Olson, 2021).

Es frecuente dividir las posiciones en dos tipos: los enfoques complejos y el enfoque simple (Noonan, 2019). Para los primeros la identidad estaría dada por alguna cualidad o propiedad, cuya continuidad garantizaría la persistencia de la misma 
persona. La posición biologista o animalista, por ejemplo, sostiene que está dada por el cuerpo: somos el mismo individuo si se da una continuidad orgánica (Olson, 1997, 2007; Blatti, 2012; Snowdon, 2014). El llamado psicologismo sostiene en cambio que la identidad consiste en la posesión de determinados rasgos mentales, como los recuerdos o el carácter (Shoemaker, 1970; Noonan, 2003). Mientras que la primera pone en segundo lugar lo mental o psíquico, intuitivamente relacionado con la identidad de la persona, la segunda se enreda en la dificultad de que una persona pasaría a ser otra al perder la memoria o cambiar su carácter. Una aproximación diferente ofrecen las teorías narrativas, que reconocen el carácter fundamental de la biografía y la relación con otras personas en la constitución de la identidad (Schechtman, 1996; Ricoeur, 1996; MacIntyre, 1984, 1989). Frente a esta y otras posturas, el llamado enfoque de la vida personal (Person Life View) sostiene que la unidad básica que garantiza la identidad, tanto sincrónica como diacrónica, estaría en el ámbito de lo práctico; de allí que no exista un único criterio para determinar la identidad, sino que apelaríamos a uno u otro según el contexto (Schechtman, 2014).

Desde Locke en adelante el debate ha sido un escenario propicio para diseñar incontables experimentos mentales que suscitan numerosas preguntas y perplejidades (Sorensen, 1992). Si tras una disección del cerebro en sus dos hemisferios surgieran dos conciencias, ¿en cuál de ellos se conservaría la misma persona? Si se uniera una conciencia a otro cuerpo -mediante un trasplante de cerebro, por ejemplo- ¿qué persona se conservaría: la correspondiente a la conciencia o a la del segundo cuerpo? En un caso de teletransportación, ¿aparecería la misma persona en el otro extremo? ¿Qué sucedería en el extremo de origen? ¿Habría que destruir ese cuerpo inicial? ¿Puede duplicarse la identidad personal? ¿Implicaría una perfecta implantación de recuerdos una especie de trasplante de la persona? La proliferación de estos casos imaginarios ha hecho que el debate parezca irresoluble, tanto que se ha vuelto clásica la postura que sostiene que el problema mismo carece de verdadera importancia (Parfit, 1984). Parecería sin embargo más razonable cuestionar y ponderar caso por caso el alcance y la relevancia de estos experimentos mentales (Johnston, 1987; Wilkes, 1988).

Para el enfoque simple (Madell, 1981, 2015; Gasser y Stefan, 2012) -que habitualmente se considera la tesis más cercana a la experiencia cotidiana y por eso a veces también de menor valor filosófico- todas las posturas mencionadas tendrían la dificultad de hacer consistir la identidad personal en algo distinto de ella misma: una cualidad, unas relaciones, una biografía o una narración, etc. Por el contrario, la identidad no sería analizable ulteriormente ni reductible a otra cosa, sino un hecho primitivo. Las otras posiciones presupondrían la identidad, pero no darían cuenta de ella y ningún criterio sería por eso suficiente. Así, por ejemplo, el cuerpo, la mente o la historia no son relevantes para mi identidad, en tanto que cuerpos, mentes e historias, sino en tanto que son "míos". Lo propio de esta persona en concreto sería indescriptible, ya que cualquier descripción se hace recurriendo a conceptos universales, por lo que podría en principio referirse a otra persona.

Otro aspecto importante del enfoque simple es que la identidad de la persona se presenta como un hecho absoluto, como una "propiedad de todo o nada", y no como algo que admite grados. Mientras que la identidad del barco de Teseo o de un club podrían admitir grados, dependiendo de la dialéctica de cambio y permanencia de aspectos materiales o formales, esto no parece trasladable a la experiencia de la identidad personal. Con el fin de explicitar esto Geoffrey Madell afirma que no tendría sentido hablar de estados mentales (como el dolor) que fueran "parcialmente míos y parcialmente no míos" (Madell, 2015, p. 8).

Al discutir la propuesta del enfoque simple es corriente señalar que no admite condiciones suficientemente informativas de la identidad, es decir condiciones que no fueran triviales ni implicaran una circularidad, un problema que afecta a la identidad en general (Olson, 2012, p. 48; Noonan, 2019). Gasser y Stefan comentan, sin embargo, que si la identidad personal fuera algo simple y por consiguiente no analizable en términos de otras propiedades, no por eso la teoría sería totalmente no informativa, ya que "el descubrimiento -si lo fuera- de que la identidad personal no es analizable es un cierto progreso en su comprensión" (Gasser y Stefan, 2012, p. 14). Apoyados en nuestra experiencia común no parece difícil decir que comprendemos la diferencia entre una persona y otra, o entre una persona y una imitación perfecta (Nida-Rümelin, 2012). Esto no quiere decir que reconocer la diferencia sea algo sencillo o que la identidad sea evidente (problemas epistémicos), sino que la identidad de ciertas características no 
sería la identidad de la persona (problema metafísico). La proximidad del enfoque simple a la experiencia cotidiana, o psicología popular (folk psychology), no implica entonces de suyo un factor invalidante, y no impide que se haya constituido en una posición sólidamente argumentada (Madell, 2015). Asimismo, aunque esta posición parecería suponer la afirmación dualista de la persona como una entidad existente de manera separada, no necesariamente es así, ya que la afirmación de un sujeto único no excluye que esté dotado de tales o cuales características.

Desde la Psicología el tema de la identidad personal fue abordado históricamente por las ramas humanísticas y existenciales. Allport (1973) lo expresa de la siguiente manera: "El sentido de la identidad del sí mismo es un
fenómeno sorprendente, puesto que el cambio es
norma inexorable del crecimiento y de la vida.
Cada experiencia origina una modificación en
nuestro cerebro, por lo que es imposible que se
produzca nuevamente una experiencia idéntica.
Por esta razón, todo pensamiento y todo acto
cambia con el tiempo. Pero la identidad del
sí mismo continúa, aunque sabemos que el
resto de nuestra personalidad ha cambiado"
(Allport, 1973, p. 146).

Dicho autor reflexiona acerca de cómo este sí mismo se va constituyendo a lo largo del ciclo vital, desde las primeras etapas del desarrollo a partir de los dos años aproximadamente. Señala también que la diferenciación del yo y el no yo está más acentuada en la cultura occidental, siendo menos marcada en otras culturas (Allport, 1973).

Por su parte Frankl (1982) expresó en sus diez tesis concernientes a la persona que la misma es un individuo, una unidad indivisible; la misma es insummabile, ya que es una totalidad y no pueden agregársele cosas; además expresa que cada persona es absolutamente un ser nuevo. Dichas características entre otras muestran el abordaje del autor en torno a la identidad personal.

En la actualidad, Moreno, Resett y Schmidt (2015) realizaron una sistematización de las conceptualizaciones del sí mismo, abordándolo como concepto clave dentro de la psicología de la persona humana. Estos autores expresan que la identidad personal está estrechamente relacionada con la personalidad. Esta identidad "es la permanencia de la conciencia de la propia personalidad en situaciones diferentes y a través del transcurso del tiempo" (Moreno et al., 2015, p. 110).

En el marco de las perspectivas filosóficas y psicológicas planteadas, nuestro estudio tuvo el objetivo de explorar un aspecto de la comprensión espontánea de la identidad del ser personal. Quisimos ver en particular si existe una percepción de que la persona no se reduce a cualidades o episodios, y cómo verbalizamos esa percepción en la experiencia ordinaria.

Finalmente, en el marco de la filosofía experimental antes mencionado existe un trabajo publicado en 2010. Shaun Nichols y Michael Bruno hicieron un test entre estudiantes de una clase de Introducción a la Filosofía en la Universidad de Arizona. Cuando preguntaban de modo abstracto la mayor parte sostenía que las características psicológicas son críticas para la persistencia del yo (self). Pero quisieron explorar la sugerencia de Bernard Williams de que las respuestas pueden cambiar significativamente de acuerdo con la manera en que están presentados los experimentos mentales. Nichols y Bruno pusieron a los participantes frente a dos escenarios en los que ellos o una tercera persona (Jerry) pasaban por una operación quirúrgica en el cerebro, luego de la cual todos sus estados mentales eran eliminados permanentemente. Como parte del tratamiento, unos días después los médicos administraban una serie de dolorosas descargas eléctricas. Una elevada proporción de los participantes, aproximadamente igual que al preguntar de manera abstracta, respondieron que ellos o Jerry sentirían el dolor de las descargas, lo que contrasta con la afirmación de que la persistencia de los aspectos psicológicos es condición de la persistencia del yo. Parecería entonces que alguna visión no-psicológi$c a$, llamada así genéricamente por contraste con la psicológica, estaría más arraigada de lo que parece en primera instancia (Nichols y Bruno, 2010). Al igual que los autores, tampoco nosotros buscamos zanjar una discusión filosófica, sino explorar las convicciones que de hecho tienen las personas.

\section{LA PROBLEMÁTICA EN LA FICCIÓN}

El estudio tuvo como punto de partida la visualización de un episodio de ficción. En los últimos años varias obras de ficción han abordado la posibilidad de emular o sustituir artificialmente a la persona humana, como por ejemplo las películas Inteligencia Artificial, I Robot, Blade Runner, Ex Machina, etc. En esta línea, una serie emblemática 
en la interacción entre lo humano, la tecnología y la ciencia ha sido Black Mirror. En el presente estudio se trabajó con el episodio "Regresa enseguida" (Be Right Back), por la nitidez con la que plantea la cuestión de la reemplazabilidad o no de la persona humana.

El capítulo narra la historia de Martha y Ash. Apenas mudados a su nueva casa, él muere en un accidente. Con un embarazo incipiente, desolada, e inducida por una amiga, Martha suscribe un servicio virtual de correo electrónico que imita las respuestas que Ash daría, en base a su información disponible en internet. Luego Martha accede a enviar los videos y audios que conserva de Ash; el programa imita entonces su voz y pueden mantener conversaciones telefónicas, recreando la interacción entre ambos. El paso decisivo ocurre cuando Martha adquiere un modelo experimental: un muñeco sintético de tamaño real con todas las características físicas de Ash, capaz de hablar y de relacionarse como una persona real. Tras la etapa de entusiasmo inicial, al darse ya cuenta de lo fantasmagórico de la situación, la protagonista dice a 'Ash': "no eres nada (...) no tienes historia (...) eres una actuación y no es suficiente". Es decir, Ash no estaba ahí, aunque la imitación buscara sustituirlo de la manera más completa posible.

Entre los temas obviamente planteados están la posibilidad de la tecnología de replicar un ser humano y los alcances de la inteligencia artificial, entre otros si esta alcanzaría eventualmente una verdadera conciencia, ya sea meramente fenoménica y sensitiva, o también más sofisticada, como sería una racional y valorativa. Se podría además pensar el capítulo bajo la perspectiva del transhumanismo (Lacerda y Ribeiro de Mattos 2020), o de cómo enfrentamos la muerte (Pérez y Genovesi 2019). Pero también resulta interesante porque nos confronta con nuestra reacción ante una imitación tendencialmente perfecta de un ser querido cuya pérdida nos ha dejado devastados. Nuestro propósito fue estudiar en qué términos afloraba la percepción del carácter irreemplazable de una persona, sugerida por el episodio.

\section{METODOLOGÍA}

\section{Participantes}

Realizamos entrevistas individuales a 10 (diez) personas con los siguientes criterios de inclusión dentro de la muestra: tener entre $25 \mathrm{y}$
46 años de edad, estar en pareja por un tiempo mínimo de 1 año (teniendo en cuenta que en la relación de pareja la identidad de la otra persona es particularmente relevante). La selección de la muestra fue no probabilística, por conveniencia y tipo bola de nieve. De las 10 personas entrevistadas, 9 eran de Argentina (provincias de Buenos Aires y Entre Ríos) y 1 era de México. Fueron 4 varones y 6 mujeres. Para evaluar el nivel socioeconómico de los participantes se utilizó el Método Graffar-Méndez Castellano (Méndez Castellano y Méndez, 1994, 2006), el que indicó que los sujetos estaban en los niveles I, II y III, lo que indica cierta homogeneidad de los niveles socioeconómicos de los participantes.

\section{Recolección de datos}

Se contactó a las personas por correo electrónico para enviarles un formulario online. Dicho formulario contenía el consentimiento informado correspondiente y un cuestionario sociodemográfico. Se les solicitó que vieran el episodio de Black Mirror "Regresa enseguida" (Be Right Back) para luego realizar una entrevista vía online. Las entrevistas se realizaron y se registraron por medio de la plataforma Zoom. Fueron entrevistas semiestructuradas, en las que se preguntaba por las impresiones acerca del capítulo, las identificaciones con los personajes y los hechos y las posibilidades de que la ciencia o la tecnología pudiera reemplazar a una persona (ver modelo de entrevista en anexo).

\section{Procedimiento de análisis de datos}

Las entrevistas fueron desgrabadas y analizadas por tres expertos en la temática, quienes siguieron los lineamientos de la Investigación Cualitativa Consensuada (CQR por sus siglas en idioma inglés) (Hill, Thompson y Williams 1997; Hill et al., 2005). En CQR los investigadores discuten los componentes de la investigación mediante preguntas abiertas para recopilar datos y usando palabras para describir fenómenos; se realiza un proceso analítico inductivo, usando un equipo y tomando decisiones por consenso, utilizando auditores y verificando resultados al verificar sistemáticamente los datos sin procesar. Los tres pasos para realizar CQR son desarrollar y codificar dominios, construir ideas centrales y desarrollar categorías para describir las consistencias entre los casos (análisis cruzado). En la presente investigación, cada experto por separado desarrolló y codificó dominios que surgían en cada 
una de las 10 entrevistas, y de esa forma luego construyó categorías. Luego con ese material se realizó un análisis cruzado para evaluar las consistencias entre las categorías surgidas en el análisis de cada uno de los expertos. Tras este análisis cruzado se obtuvieron los resultados presentados en este artículo. Si bien se utilizó CQR, no fue con la totalidad de los pasos, ya que no participaron auditores del proceso. Además, se utilizaron elementos de la Grounded Theory o Teoría Fundamentada (Strauss y Corbin, 2002), para la construcción de categorías a partir del material obtenido en las entrevistas.

\section{ANÁLISIS Y DISCUSIÓN DE LOS HALLAZGOS}

A partir del análisis realizado surgieron cuatro categorías claras en la entrevista, a saber: shock, irreemplazable, realidad-irrealidad e intuición. A continuación detallamos con ejemplos las categorías mencionadas y las articulamos con conceptos teóricos antes desarrollados.

Shock: hace referencia a una impresión negativa acerca del capítulo, con expresiones como raro, chocante, bizarro, morboso, retorcido, tétrico, enfermizo, angustiante. Ejemplos:

"muy chocante... la historia es muy chocante... más allá de la tragedia y todo eso... toda la situación de ella con el aparato, con el software... con el robot después ... es tremendamente chocante" (varón de 36 años).

Seguramente la categoría podría haberse recogido de otros episodios de la misma serie. En las conclusiones ofrecemos una posible explicación de su recurrencia en este caso particular a base de las otras categorías detectadas.

Irreemplazable: hace referencia a que no se puede reemplazar o replicar completamente a una persona con sucedáneos o imitaciones. Ejemplos:

"Porque era cierto, era solo materia, no tenía una historia esa persona, era para reemplazar su... Yo creo que ahí ella se da cuenta de que está queriendo reemplazarlo a él, y ahí ella toma conciencia de que no iba a poder hacerlo" (mujer de 32 años).

'Ash' fue visto en general como una imitación o un sucedáneo, subrayando de un modo u otro la importancia de hacer el duelo de la persona fallecida.
En un caso se habló del engaño que supone querer conservarlo todo: "( ) el gran concepto ancestral del sacrificio; sacrificas cosas para obtener otras (...) en la Modernidad no, te quedas con todo" (varón de 38 años). La respuesta sugiere una posible relación entre la idea de sacrificio, unida al carácter único de algo, y el concepto de lo insustituible, ya que la posibilidad del sucedáneo o sustituto evitaría la necesidad de sacrificar. En efecto, si nada es verdaderamente único, no tendría sentido la idea de sacrificarlo, porque siempre otra cosa podría tomar su lugar y no habría verdadera pérdida. Algo análogo sucede con la necesidad del duelo. Esta experiencia, universal por otra parte, cobra mayor importancia en el contexto de nuestra investigación porque se haría especialmente patente con la persona.

Algunos entrevistados se refirieron expresamente a la persona en términos de irreemplazable o semejantes (irreproducible, carácter único, irrepetible), pero la idea estuvo también presente de otras maneras, varias veces como la duda o la pregunta de si 'Ash' es o no es Ash. En nuestra opinión, que se haya mencionado con cierta frecuencia la cuestión de lo irreemplazable no implica que se descarte otras características como integrantes de la identidad personal, pero sí que muchos atribuyen a la persona una dimensión única, de modo que la sola replicación de características no daría lugar a la misma persona.

En un caso la respuesta a una pregunta por lo que Martha extraña de Ash fue un claro y rotundo: "A él", reafirmado luego con claridad: "lo extraña a él y extraña lo que tenían juntos" (mujer de 46 años). Aunque ponía lo más propio de la persona en su conducta, haciéndola depender en buena medida de la dotación genética, otro entrevistado dijo algo en la misma dirección: "ella quería que [el cíborg] sea como él, pero no era él" (varón de 41 años). En esta respuesta resuena una afirmación hecha en uno de los pocos estudios filosóficos conocidos por nosotros respecto del problema de la identidad personal en este episodio de Black Mirror. Su autor resume la idea diciendo: "AshBot no es Ash" (Richards, 2019, p. 43) y refiriendo al famoso texto de Nozick (1989) subraya que el nosotros generado entre Martha y Ash había sido cortado por la muerte y solo podría ser reestablecido por esas mismas dos personas.

Las respuestas sugieren no solo la no incompatibilidad, sino también un acercamiento entre el acento que pone el enfoque simple (Madell, 1981, 
2015) acerca de ese hecho primitivo que hace a la persona, y las teorías narrativas de la identidad, ya que lo absolutamente individual de una persona sería condición de una relación significativa y en el curso del tiempo.

Realidad-irrealidad: hace referencia a que la posible imitación o sucedáneo es algo que es no real, distinguiéndolo y contraponiéndolo a lo que sí es real. Ejemplos:

"Pienso que va a ser negativo para los seres humanos que se meten en relaciones de esa clase, porque en el fondo no van a ser relaciones reales" (varón de 36 años).

El tema de la realidad o no realidad de la persona y de las relaciones salió en la mayoría de las entrevistas y en distintos momentos. Sea porque el cíborg o androide no fuera una persona, sea porque fuera otra persona, faltaría realidad en la relación, sugiriendo fuertemente que la realidad misma de Ash estaría faltando también. La identidad se presenta como una propiedad de todo o nada -como señala el enfoque simple-, por lo que la relación con 'Ash' sería algo "irreal", una "apariencia".

A la pregunta por lo que más extraña Martha de Ash, un participante añadió un interesante matiz, señalando que el otro no está para satisfacerlo a uno, en lo que parece derivar la relación entre Martha y el cíborg. Si 'Ash' solo cumple una función, si solo está para satisfacer a Martha, no habría una verdadera relación, subrayando la idea de que para que esta exista hacen falta dos personas reales y el mutuo reconocimiento como tales. Y luego concluyó: "El robot está ahí para satisfacerla a ella, cuando una relación real no es así. Mi mujer no está ahí para satisfacerme a mí, mis hijas, mis amigos, mis padres, mis hermanos. Creo que me pueden hacer mejor, pero eso implica que yo también tenga que esculpirme un poco, sacar partes mías, no que solo ceda siempre el otro como sucede ahí con el robot" (varón de 36 años).

Fuera ya de la ficción, es sumamente relevante para esta cuestión el libro de Sherry Turkle, Alone Together. La autora discute ampliamente no tanto cómo la tecnología ha transformado nuestras vidas, sino lo que esperamos de ella, en particular las razones por las que muchos prefieren un robot o una imitación inanimada de una persona o de una mascota, por ejemplo. En la mayoría de los casos se trata de la búsqueda de una mejor "prestación" de la otra parte, a pesar de que la consecuencia sea quedar en soledad, como sugiere el título (Turkle 2011). Ciertamente, las preferencias son variadas en este punto y se podría pensar también en una variación de las expectativas de acuerdo con el grado de avance de la tecnología. La autora refiere muchos ejemplos y anécdotas del primer mundo tecnológico (Japón, Estados Unidos, Europa), mientras que nuestro estudio fue realizado en América Latina.

Además de la fundamental referencia a la "realidad", otras notas aparecieron para describir la presencia o compañía de la persona: la espontaneidad (con mayor frecuencia), la subjetividad, la novedad y la creatividad. Todas ellas enfatizan de diferentes modos lo propio o exclusivo de cada uno y podrían servir también como categorías descriptivas de lo que hace al carácter único de la persona.

En otro caso, aunque había defendido que no se podría replicar una persona al $100 \%$ y había dicho también que no habría dado el paso que dio Martha, a la pregunta acerca de un hipotético duplicado perfecto - una persona con exactamente las mismas características- un entrevistado respondió que "toda persona se aferraría a eso", aunque hubiera que hacer el duelo de la persona anterior. Dijo luego que lo haría "no solo con mi pareja, sino con otros familiares; lo hubiera hecho con mi papá, lo haría con mi mamá, no solo la pareja" (mujer de 37 años). En nuestra interpretación, esta contradicción reforzaría por un lado la idea de que ser consecuente con la distinción entre una persona y sus cualidades no es tan sencillo, probablemente por razones afectivas. Además, sugeriría también la idea de que el enfoque simple no compite con los enfoques complejos al mismo nivel, sino que señala una dimensión de la persona humana no alcanzada por ellos, enfocados más bien en ciertas cualidades compartibles.

Intuición: hace referencia a la percepción de algo íntimo en la persona, difícil de poner en palabras, pero que de un modo muy preciso la define como tal persona.

“...porque pienso que en el hombre hay una dimensión espiritual que no la podés reducir a la materia y lo único que podés hacer en una fábrica o en un software es construir algún dispositivo material, o en el software que no es más que una manifestación de eso, de alguna disposición material. Creo que en el hombre hay algo más que eso y en el fondo 
no lo podés imitar. No lo podés hacer... no lo podés fabricar" (varón de 36 años).

Algunos gestos de los entrevistados -como buscando las palabras, mirando en la lejanía o juntando los dedos- podrían denotar justamente el intento de hacerse con algo inasible, escurridizo, indescriptible, aunque al mismo tiempo real. En un caso se habló de una "intuición de la persona", diciendo que "sabes que hay algo, pero no puedes terminar de precisarlo" (varón de 38 años). Fue recurrente la simple pregunta retórica de si "es o no es él [Ash]", lo que indica también la percepción de que bajo la apariencia faltaba la realidad propia del mismo Ash.

El problema no parece exclusivamente epistémico, porque no se trata de averiguar cómo sabemos que es una persona o si es la misma persona, sino si hay algo real que se pierde aun cuando se retengan todas las cualidades. En la discusión filosófica del tema se distingue entre criterio y fundamento de la identidad personal para referirse respectivamente a cómo podríamos determinar que se trata de la misma persona y a aquello por lo que esa persona es la misma persona. Aunque ambas cuestiones naturalmente se entrecruzan, pueden también distinguirse. La intuición que surgió en el estudio se aproximaría a una percepción del fundamento de la identidad personal, mientras que los criterios serían indicativos de las propiedades que normalmente acompañan a una persona determinada, pero no constituyen esa identidad propiamente hablando. De no existir un fundamento, la intuición sería un engaño o una ilusión y la persona se identificaría con sus cualidades. Esa intuición parecería corresponder al carácter irreemplazable y en última instancia también a la realidad de la persona en cuanto tal persona. No entramos aquí en el tema de si tal intuición es válida o ilusoria. Simplemente nos parece relevante la sugerencia de que apunta a un fundamento de la identidad, comprensiblemente menos asible que los criterios para determinarla.

Muchos señalaron una cierta debilidad psicológica de Martha, que explicaría cómo llegó a esa situación y por qué decidió conservar finalmente al sucedáneo. En particular, salieron a relucir su soledad, su desesperación, el incipiente embarazo, la ausencia de otros vínculos como causantes de la primera decisión, y tanto su egoísmo como, en un caso, una especie de adicción a 'Ash' para explicar la decisión de conservarlo. En general, se hizo también hincapié en que originalmente la relación entre Martha y Ash no era muy buena, sobre todo en lo que a él respecta, porque muchas veces estaba como ausente, no involucrado de lleno en la relación.

Una hipótesis para estudiar conceptual y empíricamente a partir de este resultado es que Martha cede ante la posibilidad de un sucedáneo, de una imitación, justamente porque la relación no era lo suficientemente sólida. En este caso podría pensarse que la afirmación y valoración del carácter único de la otra persona sería correlativa de la robustez del vínculo, que predispondría negativamente a aceptar un sucedáneo. En otras palabras, podría estudiarse esta intuición de que, cuanto más hondo es el vínculo, más relevante sería la identidad de la persona y menos probable su reducción a mera dispensadora de determinadas prestaciones.

\section{CONCLUSIONES Y FUTURAS LÍNEAS DE INVESTIGACIÓN}

Es posible observar una conexión entre las categorías identificadas en el análisis. Sin pronunciarnos acerca de la verdad del planteo filosófico de fondo (metafísico y epistémico), proponemos una lectura interdisciplinaria posible.

Si se pensara que la persona consiste en una suma de cualidades, o que hay un criterio relativamente sencillo para identificar la presencia de una determinada persona, el problema no se presentaría como tan agudo. Pero para quien entiende que cada persona tiene un carácter único y es por consiguiente irreemplazable, es más lógico pensar en una percepción intuitiva y difícilmente descriptible de ese carácter. Los términos empleados en una descripción guardan con la identidad una relación contingente y uno a uno no parecerían ser exclusivos de una única persona. Eso explicaría también que se hable de irrealidad en la relación con una imitación, ya que al faltar eso único e irrepetible, aun en presencia de tantos rasgos semejantes o incluso idénticos, la persona misma estaría ausente. La identidad aparece como una propiedad de todo o nada. De ahí la caracterización de la situación que presenta el episodio como chocante y bizarra: lo verdaderamente esencial está faltando y la pretensión de sustituirlo solo contribuye a generar un mayor rechazo o extrañeza.

Teniendo en cuenta que ninguno de los entrevistados conocía el objetivo puntual de nuestra investigación, y a pesar de que muchas veces las preguntas fueron interpretadas más bien en la línea 
de pensar las posibilidades de la tecnología de sustituir lo humano en cuanto humano (Misseri, 2017), los resultados obtenidos sugieren la idea de que la individualidad numérica es reconocida como algo constitutivo de la persona y que esa individualidad no descansa en una característica, reproducible o recreable en otro individuo. Basados en las entrevistas realizadas podemos concluir también que esa individualidad se asocia espontáneamente con lo biográfico y que la solidez de las relaciones próximas y significativas contribuirían a hacerla patente y reconocer su importancia. Dichos resultados serían compatibles con las teorías psicológicas acerca del sí mismo que dan gran importancia a la conciencia de la personalidad en el transcurso del tiempo, resaltando el aspecto biográfico (Moreno et al., 2015). Sería interesante investigar esto último con métodos tanto cualitativos como cuantitativos.

La dificultad para captar, expresar o ser coherente con algunas ideas -por ejemplo, con la distinción entre una persona y sus cualidades- nos hizo pensar que en futuras entrevistas o estudios con grupos focales se podría presentar al final a los participantes un texto o a una serie de afirmaciones que expresen la intención del estudio. Eso podría disparar nuevas reflexiones o cambios de opinión y quizás también sacar alguna conclusión sobre las repercusiones éticas del tema.

Si bien la discusión filosófica respecto de la identidad personal se sitúa a un nivel fundamentalmente conceptual y argumentativo, y por tanto un estudio de esta naturaleza no podría zanjarlo, pensamos que sí puede arrojar alguna información útil; teniendo en cuenta además que este es un estudio interdisciplinario que ha adoptado una metodología empírica propia de la ciencia psicológica. En primer lugar, observamos que la percepción de la persona como algo único y hasta cierto punto distinguible de sus manifestaciones se da de modo bastante articulado, no como una vaga impresión, fácilmente reconducible a una ilusión. En segundo lugar, nos parece que sería un contrasentido descuidar el significado ordinario de una noción como la de persona, que a pesar de su gran riqueza denota una experiencia universal. En tercer lugar, la distinción usual en la filosofía entre los enfoques complejos y el enfoque simple de la identidad personal permite diferenciar entre características físicas y mentales, y el contenido biográfico de una vida y de una relación, por un lado, y esa intuición de lo incomunicable, por otro. De ambos habría algún conocimiento o percepción común -es decir, un correlato epistémico- y el fundamento al que hace referencia el enfoque simple de la identidad personal no sería una mera hipótesis o una entidad teórica, sino que tendría un fuerte asidero en nuestra experiencia.

Nuestro trabajo tiene ciertamente varias limitaciones. Algunas se desprenden de la trama del episodio de Black Mirror que utilizamos como disparador de las reflexiones, y que podría haber sesgado en forma negativa la valoración de la posibilidad misma del reemplazo de un ser querido. Aunque suponemos que tal sesgo no alteraría la percepción mayoritaria de que las personas amadas no son reemplazables en tanto que personas amadas, en futuras investigaciones se podría presentar el tema como un mero experimento mental, desprendido de la carga emotiva que, por fuerza, debe tener una buena ficción para resultar atractiva. De igual modo, el capítulo podría haber inducido a los participantes a hacer foco en la irreemplazabilidad del ser humano qua ser humano, y no tomado como una persona en su individualidad numérica. Es posible destacar que, si bien hay que apreciar los resultados y reflexiones realizadas a la luz de esta limitación, los mismos no dejan de ser un aporte para la investigación interdisciplinar de la identidad personal.

Finalmente, no hemos considerado la cuestión del reemplazo o sustituto de roles (pareja, padres, hijos, etc.), que ocupa un lugar intermedio, pero contiene en sí tanto lo genérico como lo individual. Este último punto ameritaría un tratamiento que, por complejidad y extensión, excede las posibilidades del presente trabajo.

Para subsanar estas limitaciones futuras investigaciones podrían seguir dos caminos contrarios: o bien proponer variaciones en la presentación del problema para que las reflexiones apunten de un modo más específico al problema de la individualidad numérica, o al de la sustitución de roles; o bien indagar acerca del tema sin ningún punto de partida específico para obtener respuestas más espontáneas. 


\section{REFERENCIAS}

Allport, G. W. (1973). La personalidad, su configuración y desarrollo. Barcelona: Herder.

Blatti, S. (2012). A new argument for animalism. Analysis, $72(4), 685-690$

Córdoba, M. (2017). Identidad personal. En C. E. Vanney, I. Silva y J. F. Franck (Eds.), Diccionario Interdisciplinar Austral. Recuperado de http://dia.austral.edu.ar/Identidad_personal

Frankl, V. (1982). La voluntad de sentido. Barcelona: Herder.

Gasser, G. y Stefan, M. (Eds). (2012). Personal Identity. Complex or Simple?. Cambridge University Press.

Hill, C. E., Knox, S., Thompson, B. J., Williams, E. N., Hess, S. A. y Ladany, N. (2005). Consensual qualitative research: An update. Journal of counseling psychology, 52 (2), 196-205.

Hill, C. E., Thompson, B. J. y Williams, E. N. (1997). A guide to conducting consensual qualitative research. The counseling psychologist, 25 (4), 517-572.

Johnston, M. (1987). Human Beings. The Journal of Philosophy, 84, 59-83.

Knobe, J. y Nichols, S. (2017). Experimental Philosophy. En E. N. Zalta (Ed.), The Stanford Encyclopedia of Philosophy (Winter 2017 Edition). Recuperado de https://plato.stanford. edu/archives/win2017/entries/experimental-philosophy

Lacerda, I. y Ribeiro de Mattos, T. (2020) Be Right Back: Humans, Artificial Intelligence and Dasein in Black Mirror, Communication, technologies et développement, 8, doi: 10.4000/ctd. 3353

MacIntyre, A. (1984). After Virtue. Notre Dame: University of Notre Dame Press.

MacIntyre, A. (1989). The Virtues, the Unity of a Human Life and the Concept of a Tradition. En S. Hauerwas y L. G. Jones (Eds.), Why Narrative? Readings in Narrative Theology (pp. 89-111). Grand Rapids, MI: W.B. Eerdmans.

Madell, G. (1981). The Identity of the Self. Edinburgh University Press.

Madell, G. (2015). The Essence of the Self. In Defense of the Simple View of Personal Identity. New York, London: Routledge.

Méndez-Castellano, H. y Méndez, M. C. (1994). Sociedad y estratificación. Método Graffar-Mendez Castellano. Caracas: Fundacredesa.

Méndez Castellano, H. y Méndez M. C. (2006). Sociedad y estratificación. Método Graffar-Méndez Castellano. Caracas: Fundación Centro de Estudios sobre Crecimiento y Desarrollo de la Población venezolana.

Misseri, L. E. (2017). Añicos de humanidad: Black Mirror y el transhumanismo, Verba Volant. Revista de Filosofía y Psicoanálisis, 7 (2), 93-107.

Moreno, J. E., Resett, A. y Schmidt, A. (2015). El sí mismo. Una noción clave de la psicología de la persona humana. Buenos Aires: EDUCA.
Muñoz Corcuera, A. (2020). Identidad personal. Enciclopedia de la Sociedad Española de Filosofía Analítica. Recuperado de http://www.sefaweb.es/identidad-personal

Nichols, S. y Bruno, M. (2010). Intuitions about personal identity: An empirical study. Philosophical Psychology, 23 (3), 293-312, doi: 10.1080/09515089.2010.490939

Nida-Rümelin, M. (2012). The non-descriptive individual nature of conscious beings. En G. Gasser y M. Stefan (Eds.), Personal Identity. Complex or Simple? (pp. 157-176). Cambridge University Press.

Noonan, H. (2003). Personal Identity. London: Routledge.

Noonan, H. (2019). Personal Identity: The Simple and Complex Views Revisited, Disputatio, 11, 9-22, doi: 10.2478/ disp-2019-0001

Nozick, R. (1989), Love's bond. En The Examined Life (pp. 6886). New York: Simon \& Schuster.

Olson, E. T. (1997). The Human Animal. Personal Identity without Psychology. Oxford: OUP.

Olson, E. T. (2007). What Are We? A Study in Personal Ontology. Oxford: OUP.

Olson, E. T. (2012). In search of the simple view. En G. Gasser y M. Stefan (Eds.), Personal Identity. Complex or Simple? (pp. 44-62). Cambridge University Press.

Olson, E. T. (2021). Personal Identity. En E. N. Zalta (Ed.), The Stanford Encyclopedia of Philosophy (Spring 2021 Edition). Recuperado de http://plato.stanford.edu/archives/spr2021/ entries/identity-personal

Parfit, D. (1984). Reasons and Persons. Oxford: Clarendon Press.

Pérez, E. y Genovesi, S. (2019). Death in Black Mirror: How Should We Deal with Our Mortality? En D. K. Johnson (Ed.), Black Mirror and Philosophy. Dark Reflections (pp. 292-300). Hoboken: Wiley Blackwell.

Perry, J. (Ed.). (2008). Personal Identity. Berkeley: University of California Press.

Plakias, A. (2015). Experimental Philosophy. En Oxford Handbooks Online, doi: 10.1093/oxfordhb/9780199935314.013.17

Ricoeur, P. (1996). Sí mismo como otro. Madrid: Siglo XXI.

Richards, B. (2019). Be Right Back and Rejecting Tragedy: Would You Bring Back Your Deceased Loved One? En D. K. Johnson (Ed.), Black Mirror and Philosophy. Dark Reflections (pp. 41-49). Hoboken: Wiley Blackwell.

Schechtman, M. (1996). The Constitution of Selves. Ithaca: Cornell University Press.

Schechtman, M. (2014). Staying Alive. Personal Identity, Practical Concerns, and the Unity of a Life. Oxford: OUP.

Shoemaker, S. (1970). Persons and Their Pasts. American Philosophical Quarterly, 7, 269-285.

Snowdon, P. (2014). Persons, Animals, Ourselves. Oxford: OUP. 
Sorensen, R. A. (1992). Thought Experiments. Oxford: OUP.

Strauss, A. y Corbin, J. (2002). Bases de la investigación cualitativa. Técnicas y procedimientos para desarrollar la teoría fundamentada. Bogotá: CONTUS-Editorial Universidad de Antioquia.
Turkle, S. (2011). Alone Together. Why We Expect More From Technology and Less From Each Other. New York: Basic Books. Wilkes, K. V. (1988). Real People. Personal Identity without Thought Experiments. Oxford: Clarendon Press. 


\section{ANEXO: MODELO DE ENTREVISTA}

1. ¿Cuándo viste el episodio?

2. ¿Cuál fue tu primera reacción o pensamiento luego de ver el episodio?

3. En algunos pasajes Martha le dice cosas intensas a Ash como en la escena del acantilado lo siguiente "no eres nada (...) no tienes historia (...) eres una actuación... y no es suficiente" ¿Por qué te parece que Martha dice a Ash esas cosas?

4. ¿Qué te parece que Martha extraña más de Ash?

5. ¿Te ves en la situación de Martha? ¿Qué harías vos? ¿Qué sentirías?
6. ¿Qué piensas de la idea de que la ciencia pueda replicar completamente a una persona?

7. Supongamos ahora que existe una persona real, no un producto de la tecnología, que tiene exactamente las mismas características físicas que tu pareja, el mismo carácter, las mismas reacciones, y que se lograra implantarle los mismos recuerdos, como si hubieran pasado la vida juntos. Es decir, si no hubiera forma de distinguirlas, salvo que te lo dijeran, ¿qué reacción tendrías?

8. Poniéndose ahora en el lugar de Ash, ¿qué te generaría saber o pensar que tu pareja actuaría como Martha? 